\title{
WHAT YOU LOOK AT IS WHAT YOU GET: EYE MOVEMENT-BASED INTERACTION TECHNIQUES
}

\author{
Robert J.K. Jacob \\ Human-Computer Interaction Lab \\ Naval Research Laboratory \\ Washington, D.C.
}

\begin{abstract}
In seeking hitherto-unused methods by which users and computers can communicate, we investigate the usefulness of eye movements as a fast and convenient auxiliary user-to-computer communication mode. The barrier to exploiting this medium has not been eye-tracking technology but the study of interaction techniques that incorporate eye movements into the user-computer dialogue in a natural and unobtrusive way. This paper discusses some of the human factors and technical considerations that arise in trying to use eye movements as an input medium, describes our approach and the first eye movement-based interaction techniques that we have devised and implemented in our laboratory, and reports our experiences and observations on them.
\end{abstract}

KEYWORDS: Eye movements, eye tracking, interaction techniques, human-computer interaction, input.

\section{INTRODUCTION}

Current user-computer dialogues tend to be onesided, with the bandwidth from the computer to the user far greater than that from user to computer. A fast and effortless mode of communication from a user to a computer would help redress this imbalance. We therefore investigate the possibility of introducing the movements of a user's eyes as an additional input medium. While the technology for measuring eye movements in real time has been improving, what is needed is appropriate interaction techniques that incorporate eye movements into the user-computer dialogue in a convenient and natural way. This paper

Permission to copy without fee all or part of this material is granted provided that the copies are not made or distributed for direct commercial advantage, the ACM copyright notice and the title of the publication and its date appear, and notice is given that copying is by permission of the Association for Computing Machinery. To copy otherwise, or to republish requires a fee and/or specific permission. discusses some of the human factors and technical considerations that arise in trying to use eye movements as an input medium, describes our approach and the first eye movement-based interaction techniques that we have devised and implemented in our laboratory, and reports our experiences and observations on them.

\section{BACKGROUND}

\section{Methods for Measuring Eye Movements}

Available techniques for measuring eye movements range from the not-quite-sublime to the almostridiculous. First, note that our goal is to measure visual line of gaze, that is, the absolute position in space at which the user's eyes are pointed, rather than, for example, the position of the eyeball in space or the relative motion of the eye within the head [14].

The simplest eye tracking technique is electronic recording, using electrodes placed on the skin around the eye to measure changes in the orientation of the potential difference that exists between the cornea and the retina. However, this method is more useful for measuring relative eye movements than absolute position. Perhaps the least user-friendly approach uses a contact lens that fits precisely over the bulge at the front of the eyeball and is held in place with a slight suction. This method is extremely accurate, but suitable only for laboratory studies. More practical methods use remote imaging of a visible feature located on the eyeball, such as the boundary between the sclera and iris, the outline of the pupil, or the corneal reflection of a light shone at the eye. All these require the head to be held absolutely stationary (a bite board is customarily used), to be sure that any measured movement represents movement of the eye, not the head. However, by tracking two features of the eye simultaneously, it is possible to distinguish head movements (the two features move together) from eye movements (the two move with respect to one another), and the head 
need not be rigidly fixed. This is currently the most practical method for use in a conventional computer-and-user setting, since the eye tracker sits several feet from the user, nothing contacts him or her, and the head need not be clamped. In our laboratory, we use an Applied Science Laboratories (Waltham, Mass.) Model 3250R eye tracker $[9,14]$. Figure 1 shows the components of this type of eye tracker. It simultaneously tracks the corneal reflection (from an infrared light shining on eye) and the outline of the pupil (illuminated by same light). Visual line of gaze is computed from the relationship between the two tracked points.

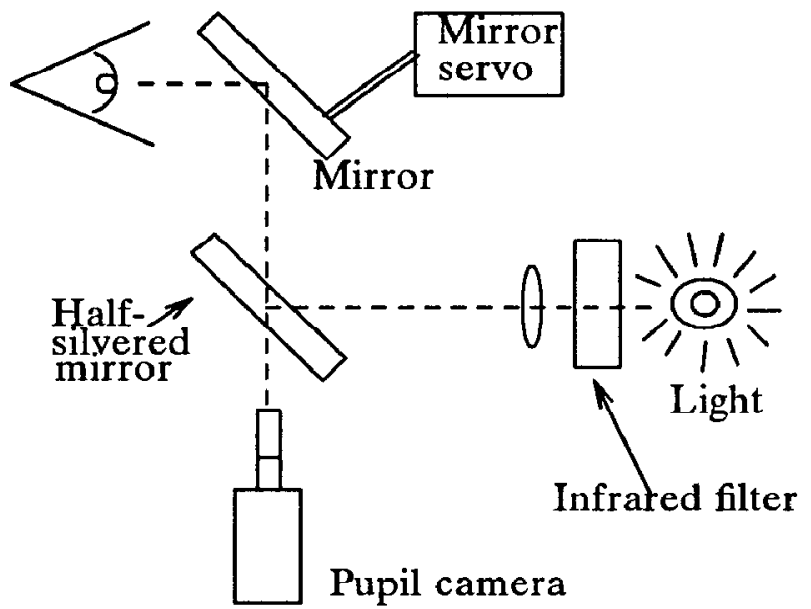

Figure 1. Illustration of components of a corneal reflection-plus-pupil eye tracker. The pupil camera and illuminator operate along the same optical axis, via a half-silvered mirror. The servo-controlled mirror is used to compensate for the user's head motions.

\section{Previous Work}

While technology for measuring visual line of gaze is adequate, there has been little research on using this information in real time. There is a considerable body of research using eye tracking, but it has concentrated on eye movement data as a tool for studying motor and cognitive processes by recording the eye movements and subsequently analyzing them $[7,10]$. Real-time eye input has been used most frequently for disabled (quadriplegic) users, who can use only their eyes for input $[4,8]$. Our interest is, instead, on dialogues that combine real-time eye movement data with other, more conventional modes of user-computer communication. Richard Bolt did some of the earliest work in this particular area and demonstrated several innovative uses of eye movements [1,2]. Floyd Glenn [5] used eye movements for several tracking tasks involving moving targets. Ware and Mikaelian [13] reported an experiment in which simple target selection and cursor positioning operations were performed substantially faster with an eye tracker than with any of the more conventional cursor positioning devices.

\section{Characteristics of Eye Movements}

To see an object clearly, it is necessary to move the eyeball so that the object appears on the fovea, a small area at the center of the retina. Because of this, a person's eye position provides a rather good indication (to within the one-degree width of the fovea) of what specific portion of the scene before him he is examining. The most common way of moving the eyes is a sudden, ballistic, and nearly instantaneous saccade. It is typically followed by a fixation, a $200-600 \mathrm{~ms}$. period of relative stability during which an object can be viewed. During a fixation, however, the eye still makes small, jittery motions, generally covering less than one degree. Smooth eye motions, less sudden than saccades, occur only in response to a moving object in the visual field. Other eye movements, such as nystagmus, vergence, and torsional rotation are relatively insignificant in a user-computer dialogue.

The overall picture of eye movements for a user sitting in front of a computer is a collection of steady (but slightly jittery) fixations connected by sudden, rapid saccades. The eyes are rarely entirely still. They move during a fixation, and they seldom remain in one fixation for long. Figure 2 shows a trace of eye movements (with jitter removed) for a user using a computer for 30 seconds. Compared to the slow and deliberate way people operate a mouse or other manual input device, eye movements careen madly about the screen. During a fixation, a user generally thinks he is looking steadily at a single object-he is not consciously aware of the small, jittery motions. This suggests that the human-computer dialogue should be constructed so that it, too, ignores those motions, since, ultimately, it should correspond to what the user thinks he is doing, rather than what his eye muscles are actually doing.

\section{"Midas Touch" Problem}

The most naive approach to using eye position as an input might be as a direct substitute for a mouse: changes in the user's line of gaze would cause the mouse cursor to move. This is an unworkable (and annoying) approach, because people are not accustomed to operating devices just by moving their eyes. They expect to be able to look at an item without having the look "mean" something. Normal visual perception requires that the eyes move about, scanning the scene before them. It is not desirable for each such move to initiate a computer command.

At first, it is empowering simply to look at what you want and have it happen. Before long, though, it becomes like the Midas Touch. Everywhere you look, another command is activated; you cannot look anywhere without issuing a command. The challenge in building a useful eye 


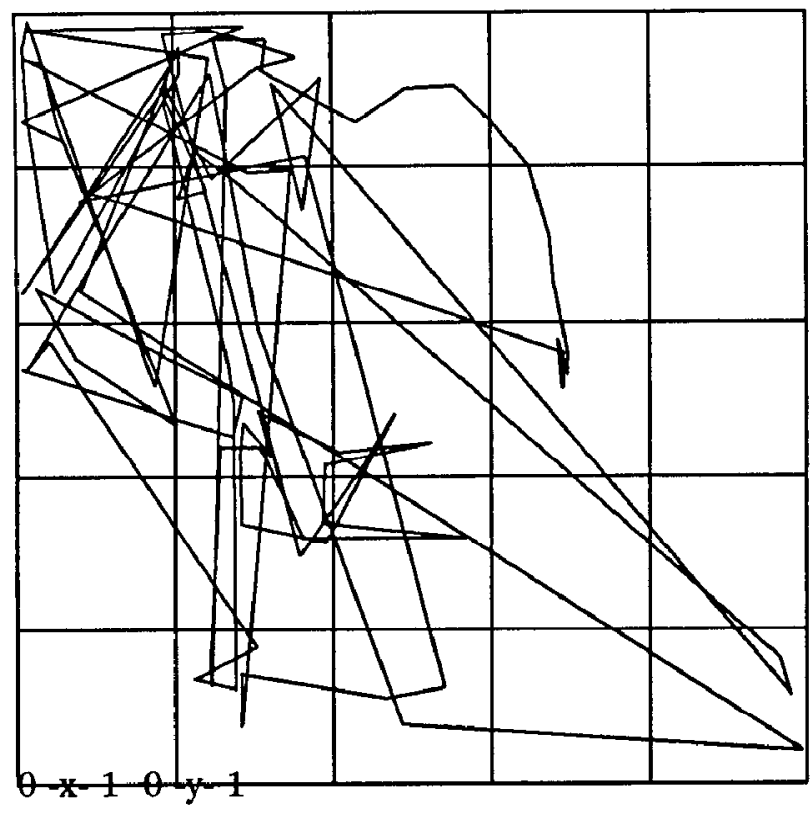

Figure 2. A trace of a computer user's eye movements over approximately 30 seconds, while performing normal work (i.e., no eye-operate interfaces) using a windowed display. Jitter within each fixation has been removed from this plot.

tracker interface is to avoid this Midas Touch problem. Ideally, the interface should act on the user's eye input when he wants it to and let him just look around when that's what he wants, but the two cases are impossible to distinguish in general. Instead, we investigate interaction techniques that address this problem in specific cases.

\section{EXPERIENCE WITH EYE MOVEMENTS}

\section{Configuration}

We use an Applied Science Laboratories corneal reflection eye tracker. The user sits at a conventional (government-issue) desk, with a Sun computer display, mouse, and keyboard, in a standard chair and office. The eye tracker camera/illuminator sits on the desk next to the monitor. Other than the illuminator box with its dim red glow, the overall setting is thus far just like that for an ordinary office computer user. In addition, the room lights are dimmed to keep the user's pupil from becoming too small. The eye tracker transmits the $x$ and $y$ coordinates for the user's visual line of gaze every $1 / 60$ second, on a serial port, to a Sun $4 / 260$ computer. The Sun performs all further processing, filtering, fixation recognition, and some additional calibration and also implements the user interfaces under study.

Observation: The eye tracker is, strictly speaking, non-intrusive and does not touch the user in any way. Our setting is almost identical to that for a user of a conventional office computer. Neverthe- less, we find it is difficult to ignore the eye tracker. It is noisy; the dimmed room lighting is unusual; the dull red light, while not annoying, is a constant reminder of the equipment; and, most significantly, the action of the servo-controlled mirror, which results in the red light following the slightest motions of user's head gives one the eerie feeling of being watched.

\section{Accuracy and Range}

A user generally need not position his eye more accurately than the width of the fovea (about one degree) to see an object sharply. Finer accuracy from an eye tracker might be needed for studying the operation of the eye muscles but is not useful for our purposes. The eye's normal jittering further limits the practical accuracy of eye tracking. It is possible to improve accuracy by averaging over a fixation, but not in a real-time interface.

Observation: Despite the mechanisms for following the user's head, we find that the steadier the user holds his head, the better the eye tracker works. We find that we can generally get two degrees accuracy quite easily, and sometimes can achieve one degree. The eye tracker should thus be viewed as having a resolution much coarser than that of a mouse or other typical devices, perhaps more like a touch screen. A further problem is that the range over which the eye can be tracked with this equipment is fairly limited. In our configuration, it can barely cover the surface of a 19 " monitor at a 24 " viewing distance.

\section{Using the Eye Tracker Data}

Our approach to processing eye movement data is to partition the problem into two stages. First we process the raw eye tracker data in order to filter noise, recognize fixations, compensate for local calibration errors, and generally try to reconstruct the user's more conscious intentions from the available information. This processing stage converts the continuous, somewhat noisy stream of raw eye position reports into tokens that are claimed to approximate more closely the user's intentions in a higher-level user-computer dialogue. Then, we design generic interaction techniques based on these tokens as inputs.

Observation: Because eye movements are so different from conventional computer inputs, we achieve success with a philosophy that tries, as much as possible, to use natural eye movements as an implicit input, rather than to train a user to move the eyes in a particular way to operate the system. We try to think of eye position more as a piece of information available to the user-computer dialogue involving a variety of input devices than as the intentional actuation of an input device. 


\section{Local Calibration}

The eye tracker calibration procedure produces a mapping that is applied uniformly to the whole screen, but we found small calibration errors appear in portions of the screen, rather than systematically across it. We introduced an additional layer of calibration into the chain, which allows the user to make local modifications to the calibration dynamically. If the user feels the eye tracker is not responding accurately in some area of the screen, he can at any point move the mouse cursor to that area, look at the cursor, and click a button.

Observation: Surprisingly, this had the effect of increasing the apparent response speed for object selection and other interaction techniques. The reason is that, if the calibration is slightly wrong in a local region and the user stares at a target in that region, the eye tracker will report the eye position somewhere slightly outside the target. If he continues to stare at it, though, his eyes will in fact jitter around to a spot that the eye tracker will report as being on the target. The effect feels as though the system is responding too slowly, but it is a problem of local calibration.

\section{Fixation Recognition}

After improving the calibration, we still observed erratic behavior in the user interface, even when the user thought he was staring perfectly still. This comes from both the normal jittery motions of the eye during fixations and from artifacts introduced when the eye tracker momentarily fails to obtain an adequate video image of the eye.

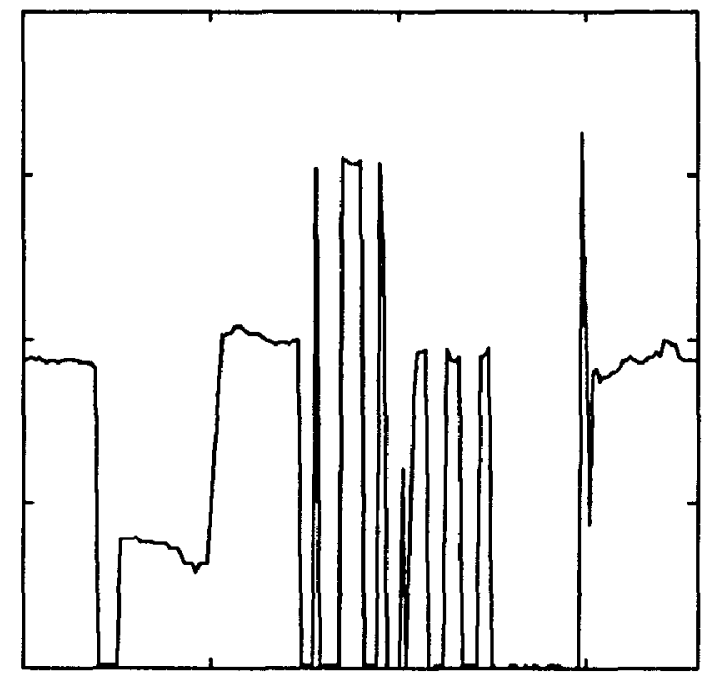

Figure 3. Illustration of erratic nature of raw data from the eye tracker. The plot shows one coordinate of eye position vs. time, over a somewhat worse-than-typical three second period.

Figure 3 shows the type of data obtained from the eye tracker. It plots the $x$ coordinate of the eye position output against time over a relatively jumpy three-second period. Zero values on the ordinate represent periods when the eye tracker could not locate the line of gaze. This might be caused by eye tracker artifacts, such as glare in the video camera, lag in compensating for head motion, or failure of the processing algorithm, or by actual user actions, such as blinks or movements outside the range of the eye tracker. During the period represented by Figure 3 , the subject thought he was simply looking around at a few different points on a CRT screen. The difference is attributable not only to the cyc tracker artifacts but to the fact that much of the fine-grained behavior of the eye muscles is not intentional. To make a reasonable input to a user-computer dialogue from the eye tracker data, we must filter out that behavior to recover the "intentional" component of the eye motions.

We return to the picture of a computer user's eye movements as a collection of jittery fixations connected by essentially instantaneous saccades. We start with an a priori model of such saccades and fixations and then attempt to recognize and quickly report the start, approximate position, and end of each recognized fixation. Blinks of up to $200 \mathrm{~ms}$. may occur during a fixation without terminating it. At first, blinks seemed to present a problem, since, obviously, we cannot obtain eye position data during a blink. However (equally obviously in retrospect), the screen need not respond to the eye during that blink period, since the user can't see it anyway. After applying this algorithm, the noisy data shown in Figure 3 are found to comprise about 6 fixations, which more accurately reflects what the user thought he was doing (rather than what his eye muscles plus the eye tracking equipment actually did). Figure 4 shows the same data, with a horizontal line marking each recognized fixation at the time and location it would be reported.

Observation: Applying the fixation recognition approach to the real-time data coming from the eye tracker yielded a significant improvement in the user-visible behavior of the interface. Filtering the data based on an a priori model of eye motion is an important step in transforming the raw eye tracker output into a user-computer dialogue.

\section{User Interface Management System}

We next turn the output of the recognition algorithm into a stream of tokens for use as input to an interactive user interface. We report tokens for eye events considered meaningful to the dialogue, much like tokens generated by mouse or keyboard events. We then multiplex the eye tokens into the same stream with those generated by the mouse and keyboard and present the overall token stream as input to our user interface management system. The desired user interface is specified to the UIMS as a collection of concurrently executing interaction objects [6]. The operation of each such object 


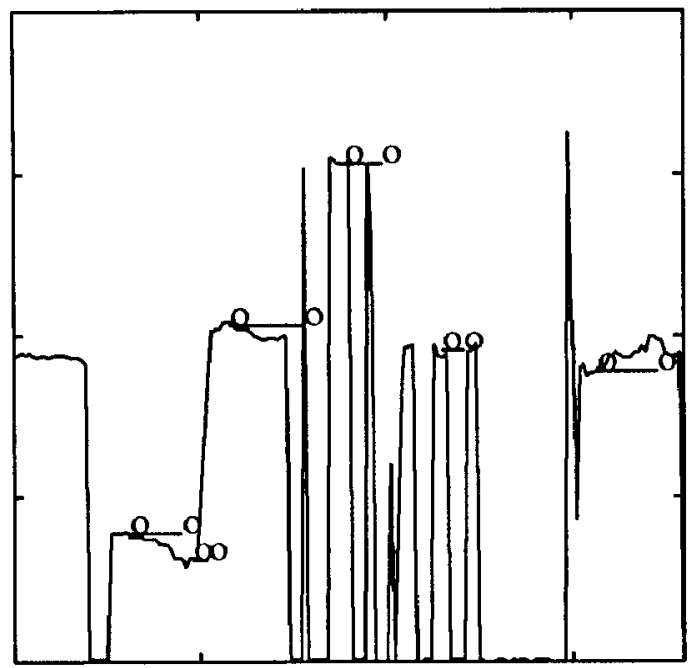

Figure 4. Result of applying the fixation recognition algorithm to the data of Figure 3. A horizontal line beginning and ending with an o marks each fixation at the time and coordinate position it would be reported.

is described by a state transition diagram that accepts the tokens as input. Each object can accept any combination of eye, mouse, and keyboard tokens, as specified in its own syntax diagram.

\section{INTERACTION TECHNIQUES}

An interaction technique is a way of using a physical input device to perform a generic task in a human-computer dialogue [11]. It represents an abstraction of some common class of interactive task, for example, choosing one of several objects shown on a display screen. This section describes the first few eye movement-based interaction techniques that we have implemented and our initial observations from using them.

\section{Object Selection}

The task here is to select one object from among several displayed on the screen, for example, one of several file icons on a desktop or, as shown in Figure 5, one of several ships on a map in a hypothetical "command and control" system. With a mouse, this is usually done by pointing at the object and then pressing a button. With the eye tracker, there is no natural counterpart of the button press. We reject using a blink for a signal because it detracts from the naturalness possible with an eye movement-based dialogue by requiring the user to think about when he or she blinks. We tested two alternatives. In one, the user looks at the desired object then presses a button on a keypad to indicate that the looked-at object is his choice. In Figure 5, the user has looked at ship "EF151" and caused it to be selected (for attribute

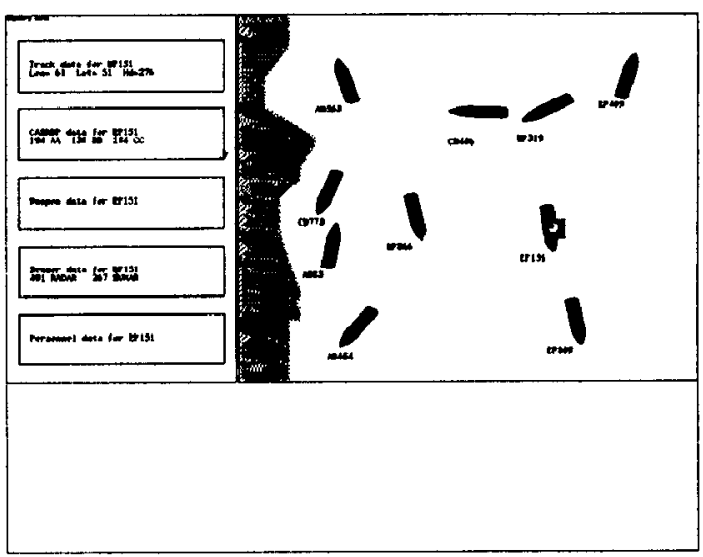

Figure 5. Display from eye tracker testbed, illustrating object selection technique. Whenever the user looks at a ship in the right window, the ship is selected and information about it is displayed in left window. The square eye icon at the right is used to show where the user's eye was pointing in these illustrations; it does not normally appear on the screen. The actual screen image uses light figures on a dark background to keep the pupil large.

display, described below). The second uses dwell time-if the user continues to look at the object for a sufficiently long time, it is selected without further operations. The two techniques can be implemented simultaneously, where the button press is optional and can be used to avoid waiting for the dwell time to expire, much as an optional menu accelerator key is used to avoid traversing a menu.

Observation: At first this seemed like a good combination. In practice, however, the dwell time approach is much more convenient. While a long dwell time might be used to ensure that an inadvertent selection will not be made by simply "looking around" on the display, this mitigates the speed advantage of using eye movements for input and also reduces the responsiveness of the interface. To reduce dwell time, we make a further distinction. If the result of selecting the wrong object can be undone trivially (selection of a wrong object followed by a selection of the right object causes no adverse effect-the second selection instantaneously overrides the first), then a very short dwell time can be used. For example, if selecting an object causes a display of information about that object to appear and the information display can be changed instantaneously, then the effect of selecting wrong objects is immediately undone as long as the user eventually reaches the right one. This approach, using a 150-250 ms. dwell time gives excellent results. The lag between eye movement and system response (required to reach the dwell time) is hardly detectable to the user, yet long enough to accumulate sufficient data for our fixation recognition and processing. The subjec- 
tive feeling is of a highly responsive system, almost as though the system is executing the user's intentions before he expresses them. For situations where selecting an object is more difficult to undo, button confirmation is used. We found no case where a long dwell time (over $3 / 4$ second) alone was useful, probably because it does not exploit natural eye movements (people do not normally fixate one spot for that long) and also creates the suspicion that the system has crashed.

\section{Continuous Attribute Display}

A good use of this object selection interaction technique is for retrieving attributes of one of the objects on a display. Our approach is to provide a separate area of the display where such attributes are always shown. In Figure 5, the window on the right is a geographic display of ships, while the text window on the left shows some attributes of one of the ships, the one selected by the user's eye movement. The idea behind this is that the user can look around the ship window as desired. Whenever he looks over to the text window, he will always find there the attribute display for the last ship looked at-presumably the one he is interested in. (The ship remains selected when he looks away from the ship window to the text window.) However, if he simply looks at the ship window and never looks at the text area, he need not be concerned that his eye movements are causing commands in the text window. The text window is double-buffered, so that changes in its contents could hardly be seen unless the user were looking directly at it at the time it changed (which, of course, he is not-he must be looking at the ship window to effect a change).

\section{Moving an Object}

We experimented with two methods for moving an object on the display. Our initial notion was that, in a direct manipulation system, a mouse is typically used for two distinct operations-selecting an object to be manipulated and performing the manipulation. The two functions could be separated and each assigned to an appropriate input device. In particular, the selection could be performed by eye position, while the hand input device is devoted exclusively to the manipulations. We therefore implemented a technique whereby the eye selects an object (ship) to be manipulated (moved on the map, in this case) and then the mouse is used to move it. The eye selection is made as described above. Then, the user grabs the mouse, presses a button, drags the mouse in the direction the object is to be moved, and releases the button. There is no visible mouse cursor, and the mouse is used as a relative position device-it starts moving from wherever the eyeselected ship was. Our second approach used the eye to select and drag the ship, and a pushbutton to pick it up and put it down. The user selects a ship, then presses a button; while the button is depressed, the ship drags along with the user's eye. When it is released, the ship remains in its new position. Since the processing described previously is performed on the eye movements, the ship actually jumps to each fixation after about $100 \mathrm{~ms}$. and then remains steadily there-despite actual eye jitter-until the next fixation.

Observation: Our initial guess was that the second method would be difficult to use: eye movements are fine for selecting an object, but picking it up and having it jump around on the screen in response to eye movements would be annoying-a mouse would give more concrete control. Once again, our guess was not borne out. While the eye-to-select/mouse-to-drag method worked well, the user was quickly spoiled by the eye-only method. Once you begin to expect the system to know where you are looking, the mouse-to-drag operation seems awkward and slow. After looking at the desired ship and pressing the "pick up" button, the natural thing to do is to look at where you are planning to move the ship. At this point, you feel, "I'm looking right at the destination I want, why do I now have to go get the mouse to drag the ship over here?" With eye movements processed to suppress jitter and respond only to recognized fixations, the motion of the dragging ship is reasonably smooth and predictable and yet appears subjectively instantaneous. It works best when the destination of the move is a recognizable feature on the screen (another ship, a harbor on a map); when the destination is an arbitrary blank spot, it is more difficult to make your eye look at it, as the eye is always drawn to features.

\section{Eye-controlled Scrolling Text}

A window of text is shown, but not all of the material to be displayed can fit. As shown at the bottom left of Figure 6, arrows appear below the last line of the text and above the first line, indicating that there is additional material not shown. If the user looks at an arrow, the text itself starts to scroll. Note, though, that it never scrolls when the user is actually reading the text (rather than looking at the arrow). The assumption is that, as soon as the text starts scrolling, the user's eye will be drawn to the moving display and away from the arrow, which will stop the scrolling. The user can thus read down to end of the window, then, after he finishes reading the last line, look slightly below it, at the arrow, in order to retrieve the next part of the text. The arrow is visible above and/or below text display only when there is additional scrollable material in that dircction.

\section{Menu Commands}

Since pop-up menus inherently assume a button, we experimented with an eye-operated pull-down menu. In Figure 7, if the user looks at the header of a pull-down menu for a given dwell time (400 ms.), the body of the menu will appear on the 


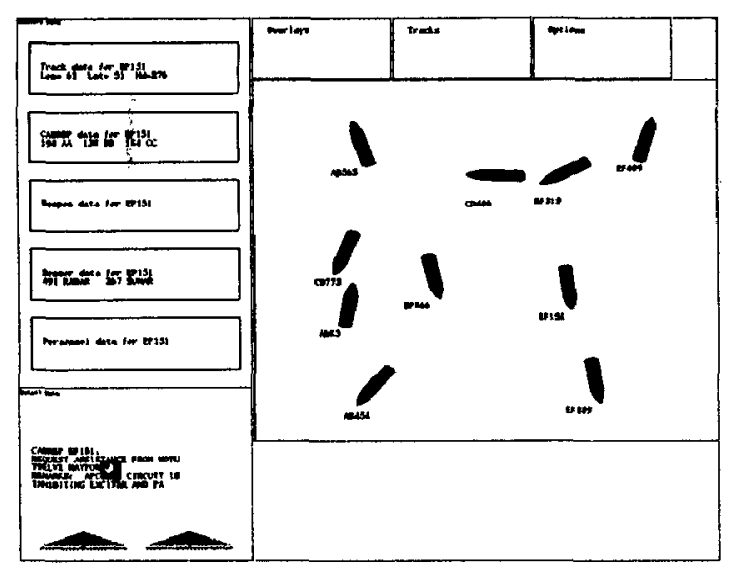

Figure 6. Another display from the testbed, showing the scrolling text and other windows.

screen. Next, he can look at the items shown on the menu. After a brief look at an item (100 ms.), it will be highlighted, but its command will not yet be executed. This allows the user time to examine the different items on the menu. If the user looks at one item for a much longer time (1 sec.), its command will be executed and the menu erased. Alternatively, once the item is highlighted, pressing a button will execute it immediately and erase the menu. If the user looks outside the menu (for $600 \mathrm{~ms}$.), the menu is erased without any command executed.

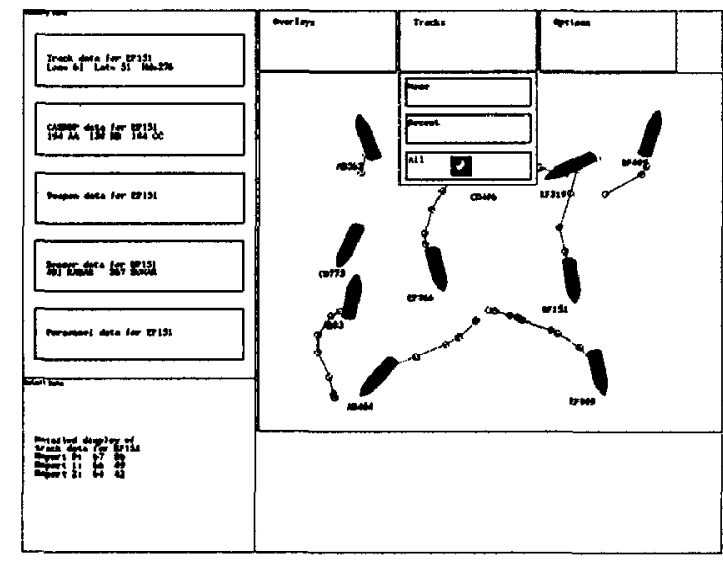

Figure 7. Testbed display showing eyecontrolled pull-down menu.

Observation: Our initial experience with this interaction technique suggests that the button is more convenient than the long dwell time for executing a menu command. This is because the dwell time necessary before executing a command must be kept quite high, at least noticeably longer than the time required to read an unfamiliar item. This is longer than people normally fixatc on onc spot, so selecting such an item requires an unnatural sort of "stare." Pulling the menu down and selecting an item to be highlighted are both done very effectively with short dwell times, as with object selection.

\section{Listener Window}

In a window system, the user must designate the active or "listener" window, that is, the one that receives keyboard inputs. Current systems use an explicit mouse command to designate the active window: simply pointing or else pointing and clicking. Instead, we use eye position-the listener window is simply the one the user is looking at. A delay is built into the system, so that user can look briefly at other windows without changing the listener window designation. Fine cursor motions within a window are still handled with the mouse, which gives an appropriate partition of tasks between eye tracker and mouse, analogous to that between speech and mouse used by Schmandt [12]. A possible extension to this approach is for each window to remember the location of the mouse cursor within it when the user last left that window. When the window is reactivated (by looking at it), the mouse cursor is restored to that remembered position.

\section{EXPERIMENTAL PLANS}

The next step in this study is to perform more controlled observations on the new techniques. Our first experiment will compare object selection by dwell time with conventional selection by mouse pick. The extraneous details of the ship display are removed for this purpose, and a simple abstract display of circular targets is used, as shown in Figure 8. In the experiment, one of the targets will be designated, and the subject's task is to find it and select it, either by eye with dwell time or mouse. Response time for the two methods will be compared. (Initial pilot runs of this procedure suggest a 30 per cent decrease in time for the eye over the mouse, although the eye trials show more variability.)

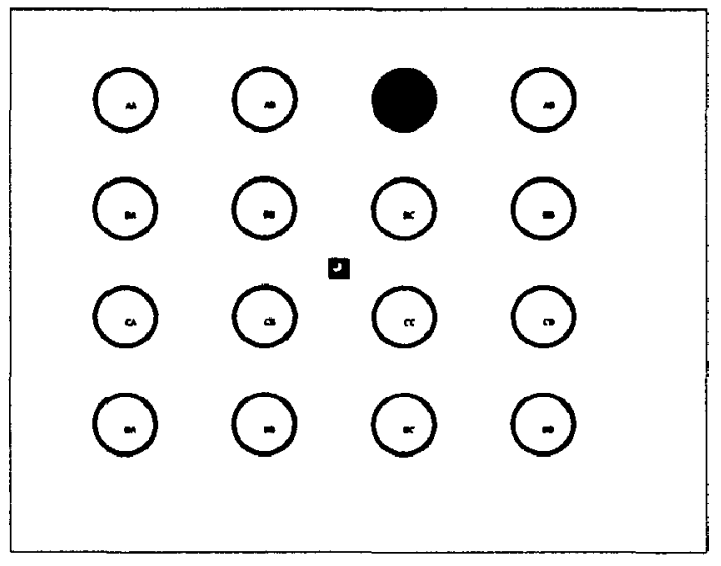

Figure 8. Display for experimental study of the object selection interaction technique. Item "AC" near the upper right has just become highlighted, and the user must now select it (by eye or mouse). 


\section{CONCLUSIONS}

We present, in Brooks' taxonomy [3], "observations" rather than "findings" of our research:

An eye tracker as an input device is far from "perfect," in the sense that a mouse or keyboard is, and that is caused both by the limitations of current equipment and, more importantly, by the nature of human eye movements. Accuracy obtainable is more similar to a touch screen than a mouse, and the range can barely cover a single CRT display. The equipment, while non-intrusive and non-contacting is still difficult to ignore. Nevertheless, it is perhaps amazing that this can be done at all; and, when the system is working wcll, it can give the powerful impression of responding to its user's intentions rather than his explicit inputs.

To achieve this, our overall approach in designing interaction techniques is, wherever possible, to obtain information from a user's natural eye movements while viewing the screen rather than requiring the user to make specific eye movements to actuate the system. We also found it important to search for and recognize fixations in the raw eye tracker data stream and construct our dialogue around these higher-level events.

In our initial interaction techniques, we observed the value of short dwell time eye-only object selection for cases where a wrong pick immediately followed by a correct pick is acceptable. For moving an object we found filtered eye movements surprisingly effective, even though a mouse initially seemed more appropriate for this task. For menu commands, we found the eye alone appropriate for popping up a menu or tentatively choosing an item, but executing an item requires a button for confirmation rather than a long dwell time.

\section{ACKNOWLEDGMENTS}

I want to thank my colleagues, Connie Heitmeyer, Preston Mullen, Linda Sibert, Stan Wilson, and Diane Zimmerman, for all kinds of help with this research.

\section{REFERENCES}

1. R.A. Bolt, "Gaze-Orchestrated Dynamic Windows," Computer Graphics 15(3) pp. 109-119 (August 1981).

2. R.A. Bolt, "Eyes at the Interface," Prcc. ACM Human Factors in Computer Systems Conference pp. 360-362 (1982).

3. F.P. Brooks, "Grasping Reality Through Illusion-Interactive Graphics Serving Science," Proc. ACM CHI'88 Human Factors in Computing Systems Conference pp. 1-11 (1988).
4. J. Cosco, "TV, Paralysis Victim Will See Eye-to-Eye," Virginian-Pilot pp. A1-A12 (October 23, 1986).

5. F.A. Glenn and others, "Eye-voicecontrolled Interface," Proc. 30th Annual Meeting of the Human Factors Society pp. 322-326, Santa Monica, Calif. (1986).

6. R.J.K. Jacob, "A Specification Language for Direct Manipulation User Interfaces," $A C M$ Transactions on Graphics 5 pp. 283-317 (1986). Special Issue on User Interface Software.

7. M.A. Just and P.A. Carpenter, "A Theory of Reading: From Eye Fixations to Comprehension," Psychological Review 87 pp. 329-354 (1980).

8. J.L. Levine, "An Eye-Controlled Computer," manuscript, IBM Thomas J. Watson Research Center, Yorktown Heights, N.Y. (1981).

9. J. Merchant, R. Morrissette, and J.L. Porterfield, "Remote Measurement of Eye Direction Allowing Subject Motion Over One Cubic Foot of Space," IEEE Trans. on Biomedical Engineering BME-21 pp. 309-317 (1974).

10. R.A. Monty and J.W. Senders, Eye Movements and Psychological Processes, Lawrence Erlbaum, Hillsdale, N.J. (1976).

11. B.A. Myers, "User-interface Tools: Introduction and Survey," IEEE Software 6(1) pp. 15-23 (1989).

12. C. Schmandt and D. Hindus, "Augmenting a Window Manager with Speech Input," Manuscript, M.I.T. Media Lab, Cambridge, Mass. (1989).

13. C. Ware and H.T. Mikaelian, "An Evaluation of an Eye Tracker as a Device for Computer Input," Proc. ACM CHI+GI'87 Human Factors in Computing Systems Conference pp. 183-188 (1987).

14. L.R. Young and D. Sheena, "Survey of Eye Movement Recording Methods," Behavior Research Methods and Instrumentation $7 \mathrm{pp}$. 397-429 (1975). 